\title{
Numerical Methods for the Approximation of Path Integrals Arising in Quantum Statistical Mechanics
}

\author{
Steve D. Bond \\ Departments of Chemistry and Mathematics, University of California - San Diego, La \\ Jolla, CA 92093, USA \\ bondQucsd.edu
}

\begin{abstract}
Discretizations of the Feynman-Kac path integral representation of the quantum mechanical density matrix are investigated. Each infinite-dimensional path integral is approximated by a Riemann integral over a finite-dimensional Sobolev space, by restricting the integration to a subspace of all admissible paths. Using this process, a wide class of methods can be derived, with each method corresponding to a different choice for the approximating subspace. The traditional "short-time" approximation and "Fourier discretization" can be recovered from this approach, using linear and spectral basis functions respectively. As an illustration, a new method is formulated using cubic elements and is shown to have improved convergence properties when applied to model problems. Experimental results will be discussed, including a double-well model and cluster simulations using the Blue Horizon.
\end{abstract}

